\title{
NUCLEOTIDES AND ORGANOPHOSPHATES OF CARDIAC, FAST AND SLOW MUSCLES OF CHICK DURING DEVELOPMENT
}

\author{
E. RADHA and R. V. KRISHNAMOORTHY* \\ Department of Zoology, Bangalore University, Bangalore 560001, India
}

(Received 18 December 1972)

\begin{abstract}
Nucleotides like ATP, ADP, AMP and organophosphate, a compound-like creatine phosphate, creatine and creatinine were estimated in the cardiac, slow anterior latissimus dorsi (ALD) and fast posterior latissimus dorsi (PLD) of the prenatal chick.

2. A decrease in the ATP level and an increase in the ADP/ATP ratio was observed with the advance in development in all muscle types.

3. The increase in ADP level with an increase in development was noted with a complementary increase in the activity of ATP creatine transphosphorylase in all types.

4. A gradual increase in creatine phosphate and a decrease in free phosphate in all the muscle types was recorded.

5. The utilization of ATP as indicated by phosphate potential was suggested to be existing during development by way of increased transphosphorylation. The heart and ALD muscles exhibited similar trends in ATP utilization up to the sixteenth day.

6. The $\mathrm{Ca}^{2+}$ and $\mathrm{Mg}^{2+}$ activated myofibrillar activity exhibited a decrease with reference to development.

7. The studies on ocular shortening of glycerinated fibres revealed that the contractility of the fibres progressively increased with development. The ALD fibres are bound to $\mathrm{Ca}^{2+}$ more than that of PLD and heart.

8. It is shown that the sensitivity of $\mathrm{Ca}^{2+}$ to the fibres changes with development as the PLD fibres become more sensitive, heart fibres lose their sensitivity and ALD fibres have a mixed nature with reference to $\mathrm{Ca}^{2+}$ sensitivity.
\end{abstract}

\section{INTRODUCTION}

DIFFERENCES in functional characteristics of fast and slow muscles are well known (Barany, 1967; Hess, 1970). However, the biochemical mechanism underlying these differences, during their origin, is little understood. Hence the present investigation has been undertaken to study the levels of nucleotides and organic phosphates in the developing chick muscles.

* Present address: Department of Zoology, University of Agricultural Sciences, Hebbal, Bangalore 560024, India. 


\section{MATERIALS AND METHODS}

The eggs of White Leghorns, Gallus domesticus (purchased from the State Poultry Farm, Hasserghatta), were set for incubation every fourth day to obtain 8-day, 12-day, 16-day and 20-day-old embryos at the same time. The eggs were incubated in electrically operated automatic incubators which maintain a temperature of $99-100^{\circ} \mathrm{F}\left(38^{\circ} \mathrm{C}\right)$ and a humidity of $55 \%$. The mortality of the embryos varied between 3 and 5 per cent. However, care has been taken to assess the age of the embryo by notifying the time of loading of the freshly laid eggs into the incubators and the time of dissecting the embryo.

The three muscles selected for the study were:

(1) the anterior latissimus dorsi (ALD in text, A in figures);

(2) the posterior latissimus dorsi (PLD in text, $\mathrm{P}$ in figures); and

(3) the cardiac muscle ( $\mathrm{H}$ in text and figure).

Muscles were isolated and immediately transferred on to the ice-bath. Generally, muscles were pooled from fifty to sixty eggs for analysis, the number of eggs used for the early developmental stages were more compared to the later stages so as to obtain a sufficient quantity of the tissue for biochemical analysis.

The adenine compounds, adenylic acid ADP and ATP, in the muscles were extracted by filtering the perchloric acid extracts $(5 \%)$ through a Dowex $200 \times \mathrm{G}$ mesh using columns $0.5 \times 10 \mathrm{~cm}$ in size as described by Strehler \& Totter (Glick, 1954). The eluents of this ionexchange resin were assayed spectrophotometrically for the nucleotide fraction concerned at $260 \mathrm{~nm}$. A Beckman DU2 spectrophotometer equipped with $1-\mathrm{cm}$ silica cells was used for this purpose. The pure nucleotides mentioned above were purchased from Sigma Chemical Co., St. Louis, Missouri, and standard curves were prepared for calibration. An unkown concentration of the nucleotide fraction was read by using the calibrated standard graph. The nucleotides in the fraction were also estimated colorimetrically (Davis $\&$ Harris, 1963) using a Beckman DU2 Spectrophotometer.

Creatine phosphate was extracted and purified by barium chloride precipitation (Ennor, 1957). The purified creatine phosphate was determined colorimetrically by estimating the acid-labile inorganic phosphorus by the method of Fiske \& Subba Row (1925).

The inorganic phosphate $\left(\mathrm{P}_{\mathrm{i}}\right)$ was estimated by the above method in aqueous extracts of muscles. In order to obtain this, the crude homogenate was centrifuged in distilled water and the clear supernatant was deproteinized with cold 5 per cent TCA and used for the assay.

The procedure for creatine determination was based on the colour reaction with diacetyl and $\alpha$-naphthol according to Ennor \& Rosenberg (1952). The creatine used for the standard was purchased from the Merck Co., Germany. Creatinine was estimated in the deproteinized extracts colorimetrically using alkaline picrate (Oser, 1965). Creatine transphosphorylase of these muscle extracts was determined according to the method of Noda et al. (1955).

The glycerinated fibres were obtained by subjecting these muscles in a stretched condition to long-term glycerol extraction. About $1 \mathrm{~g}$ of muscle was extracted with $100 \mathrm{ml}$ of $85 \%$ glycerol. Glycerol was changed twice a week for about a month after which the fibres were stored for 8 months at $0^{\circ} \mathrm{C}$. For the measurement of ocular shortening and ocular relaxation, teased bits of glycerol fibres were used. With a glass needle the extracted fibres were split longitudinally into fibre threads and mounted on the microscopic slides. Glycerol from the fibres was removed with filter paper. With an ocular micrometer changes in the length of the fibre were measured when it was treated with solutions such as: (1) $1 \mathrm{mM}$ ATP and $0 \cdot 1 \mathrm{M} \mathrm{KCl}$ and $10 \mathrm{mM} \mathrm{CaCl}_{2}$, (2) $1 \mathrm{mM} \mathrm{ATP}$ and $0 \cdot 1 \mathrm{M} \mathrm{KCl}$.

Myofibrils were obtained by the method of Perry $(1951,1952)$. The ATPase activity of the myofibrils was estimated according to Perry (1955) and the protein content by the biuret method (Layne, 1957). 
RESULTS

Figure 1 shows the changes in ATP levels in the muscles during development. In ALD there is a progressive decrease in ATP levels with an increase in the developmental period. In PLD a more or less steady ATP level is maintained up to the sixteenth day and thereafter a slight decrease is seen. Contrary to the above, the heart shows a rising phase in ATP concentration up to the twelfth day and afterwards establishes a uniform decrease. As a result, at all stages of development the ATP levels are not uniform in these three muscles. Up to the twelfth day the ALD possesses a high ATP concentration and the heart shows the lowest. The PLD at later stages maintains a higher ATP level than the heart and ALD.

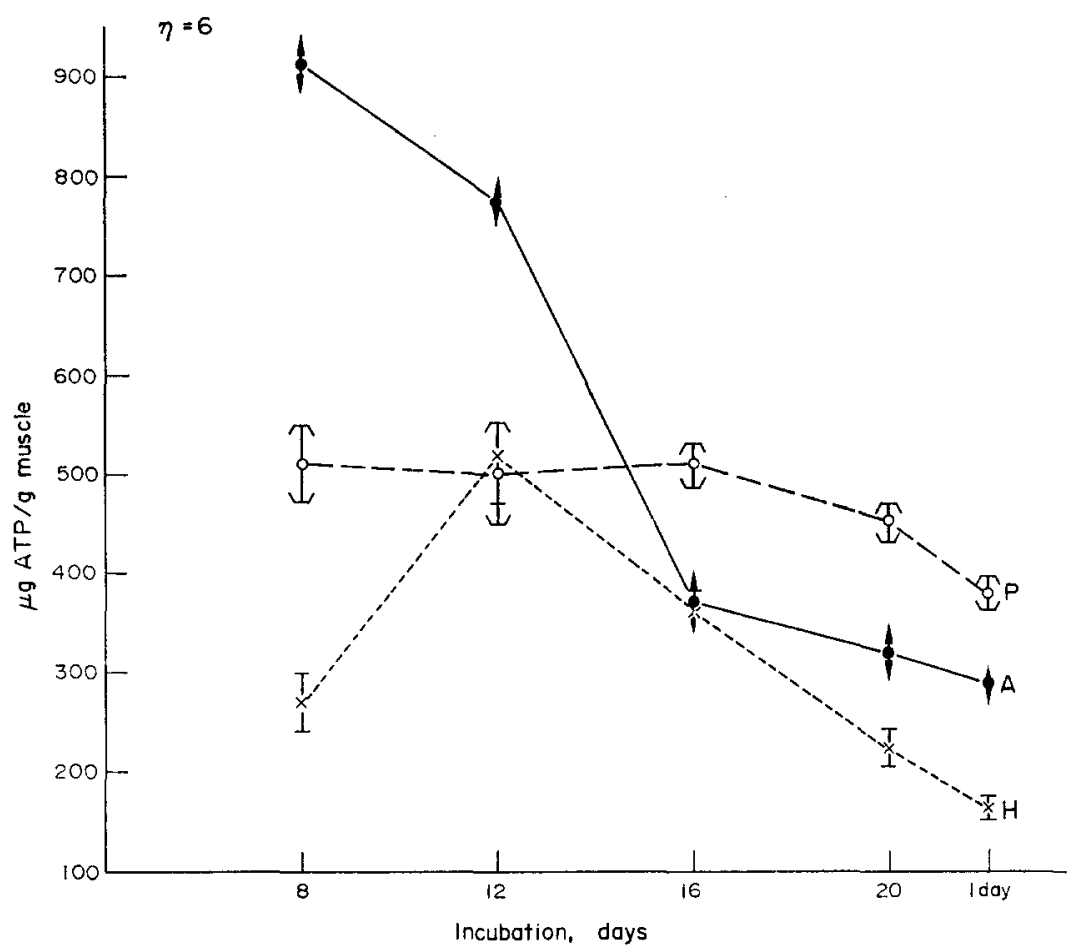

FIg. 1. Changes in the A'TP content (expressed per unit wet wt.) in the developing muscles of the chick.

The free inorganic phosphate $\left(\mathrm{P}_{\mathrm{i}}\right)$ (Fig. 2) concentration followed the same general trend as those of ATP; after the sixteenth day, the $P_{i}$ levels in ALD and PLD are more or less the same but initially the PLD shows more inorganic phosphate than does ALD. The inorganic phosphate level of ALD remains steady throughout the incubation period. On the other hand, in the heart there is a gradual decrease of inorganic phosphate. The $P_{i}$ level is known to have an impact on the contractile mechanisms of the muscle (Ruegg et al., 1971). 


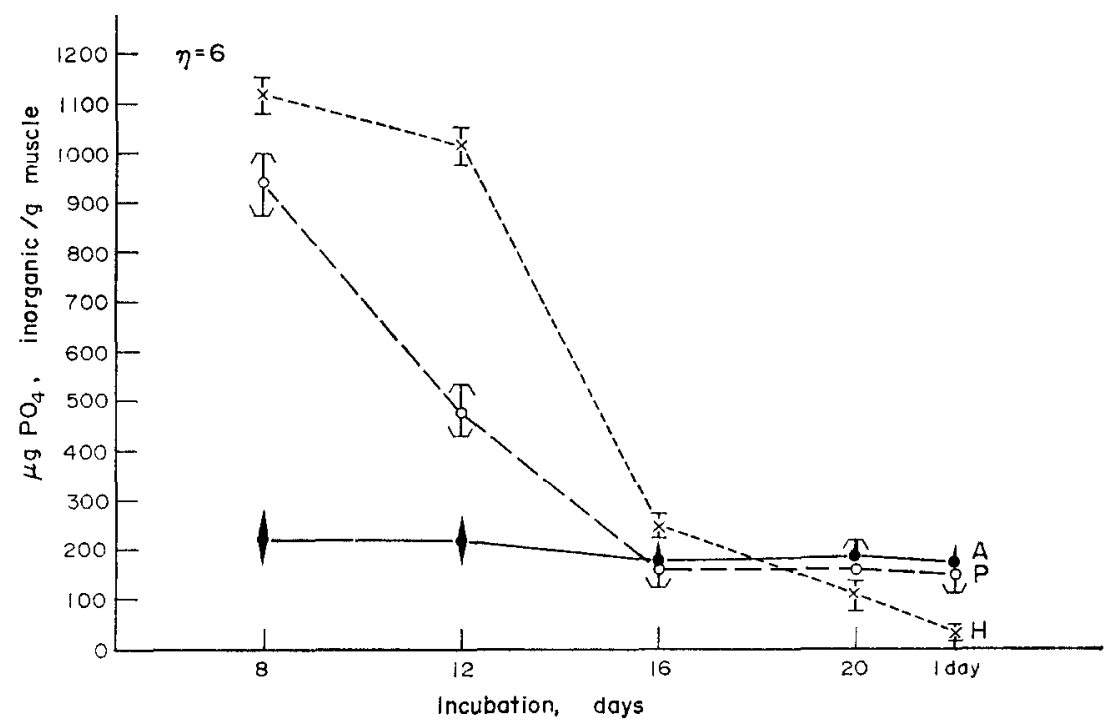

Fig. 2. Changes in the inorganic phosphate $\left(P_{i}\right)$ content (expressed per unit wet wt.) in the developing muscles of the chick.

These results suggest that the muscles show a different degree of phosphorylative and dephosphorylative mechanisms. To establish the changes in such mechanisms the levels of ADP, AMP and adenine compounds were surveyed. Figure 3

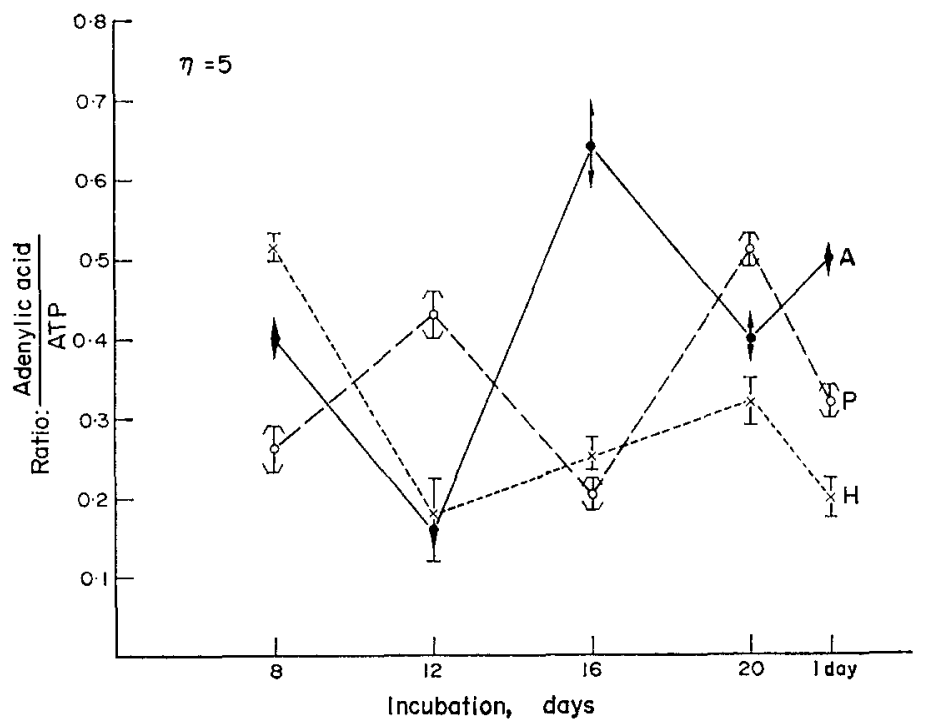

FIG. 3. Relative changes in the adenylic acid (AMP) and ATP contents of the muscle during prenatal development of the chick. Higher ratios indicate a greater AMP content relative to that of ATP. 
shows variations in the ratios of adenylic acid in the muscles during the developmental stages. Greater ratios indicate a greater concentration of adenylic acid with reference to ATP. The patterns obtained are irregular but the pattern in the changes of these ratios is opposite to that of PLD. The pattern of change in all the three muscles indicate an alternate increase or decrease with reference to the developmental periods. Higher ratios indicate a greater degree of dephosphorylation. Based on these facts it is inferred that ALD and PLD show differing degrees of dephosphorylations with varying stages of development. Adenine compounds are increased in ALD and PLD up to the twentieth day and are decreased on the first day after hatching (Fig. 4). Greater levels of adenine compounds indicate higher rates of dephosphorylation or nucleotide metabolism. In contrast to the

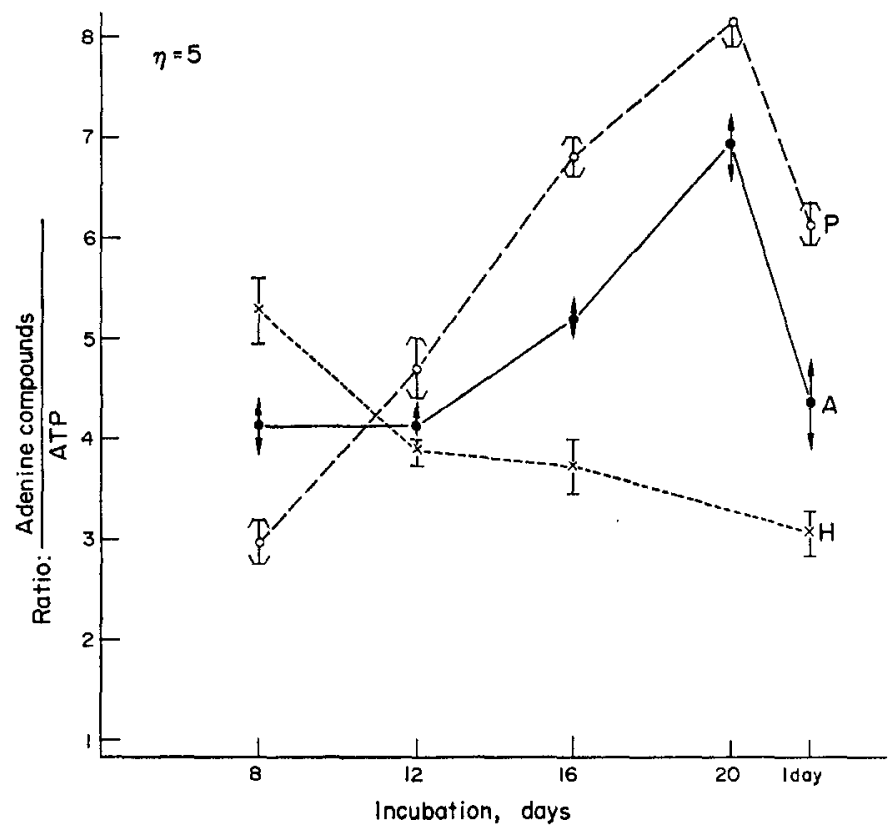

Fig. 4. Relative changes in the total adenine compounds and ATP content of the muscle during prenatal development of the chick. Higher ratios indicate a greater amount of adenine compounds.

skeletal muscle the heart maintains a negative trend in the levels of adenine compounds. In other words, in the heart muscle the nucleotide metabolism shows a reduced rate with an increase in the developmental period.

The ADP levels as shown by ADP/ATP ratios (Fig. 5) increase in all the three muscles with an increase in the days of development. The PLD sharply increases in the ADP levels up to the twentieth day of incubation and afterwards shows a slight decrease, but the heart and ALD constantly show an increase in the ADP level. 


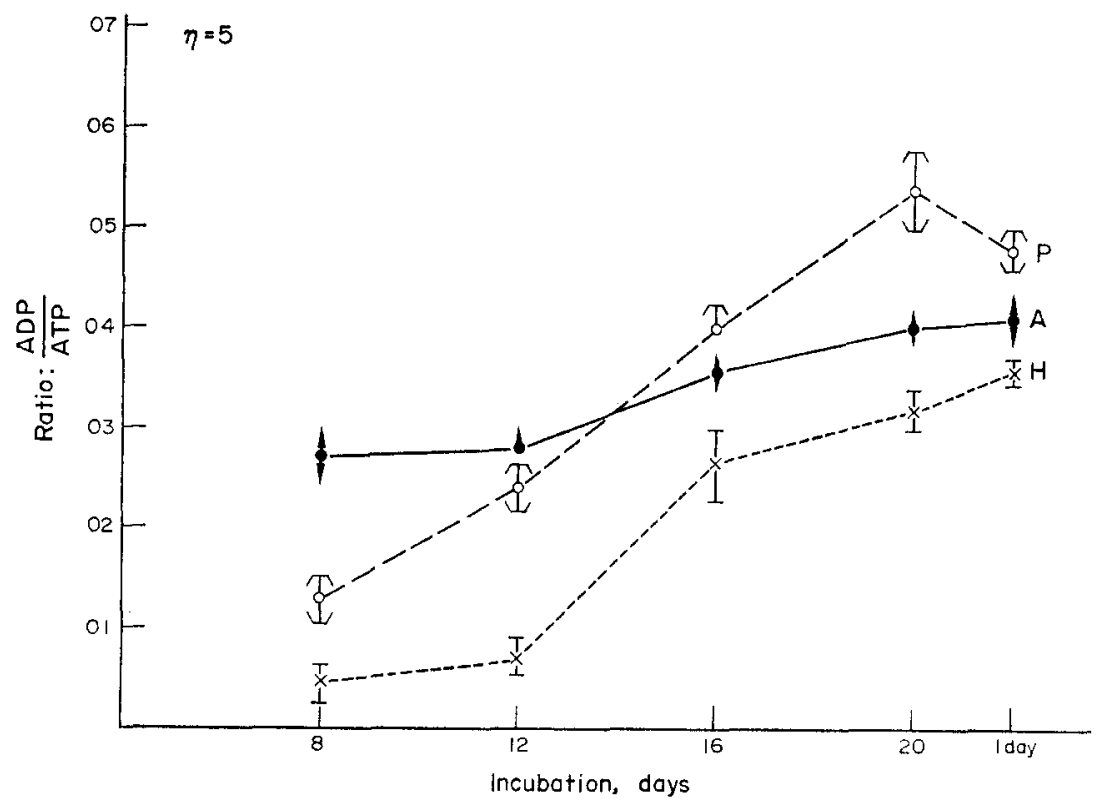

FIG. 5. Relative changes in the ADP and ATP levels of the muscles during prenatal development of the chick. Greater ratios indicate greater ADP levels.

Comparing the trends for the adenine compounds, ATP and inorganic phosphates, the increase in ADP levels clearly substantiate the nucleotide (ATP) breakdown at later stages of development. Since adenine compounds increase in the same way as ATP in the skeletal muscles it is suggested that ATP undergoes dephosphorylation to a greater degree. The negative steps in the ratio of adenine compounds as well as ATP indicate the rephosphorylation mechanisms. The opposite trends obtained in the case of the heart muscle are suggestive of a lower degree of dephosphorylation.

Figure 6 illustrates the creatine phosphate levels in the muscle during development. As is evident from this figure the skeletal muscles ALD and PLD gradually increase in creatine phosphate (CP) levels, whereas the heart depletes with increase in the stages of development. The ALD yields more CP than PLD at any stage of incubation. In the heart, the CP level increases up to the twelfth day and later falls gradually and maintains a steady state between the twentieth and first day after hatching.

Creatinine is the anhydride of creatine and is apparently the result of some special processes of normal metabolism which takes place at a constant rate and involves muscle creatine (Cantarow \& Schepartz, 1967). CP is also converted by the loss of $\mathrm{H}_{3} \mathrm{PO}_{4}$ into creatinine. Creatinine is excreted in mammals through their urine and is said to increase in conditions associated with muscular atrophy and dystrophy (Milhorat, 1967; Guth, 1969). 


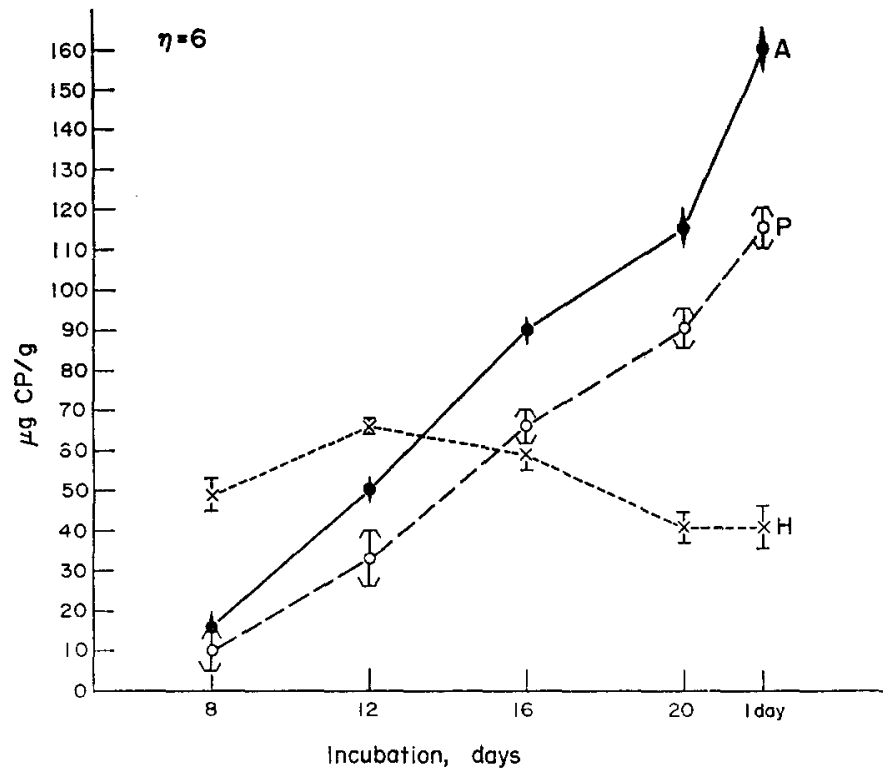

Fig. 6. Changes in the creatine phosphate levels (expressed per unit wet wt.) in the muscles during prenatal development of chicken muscles.

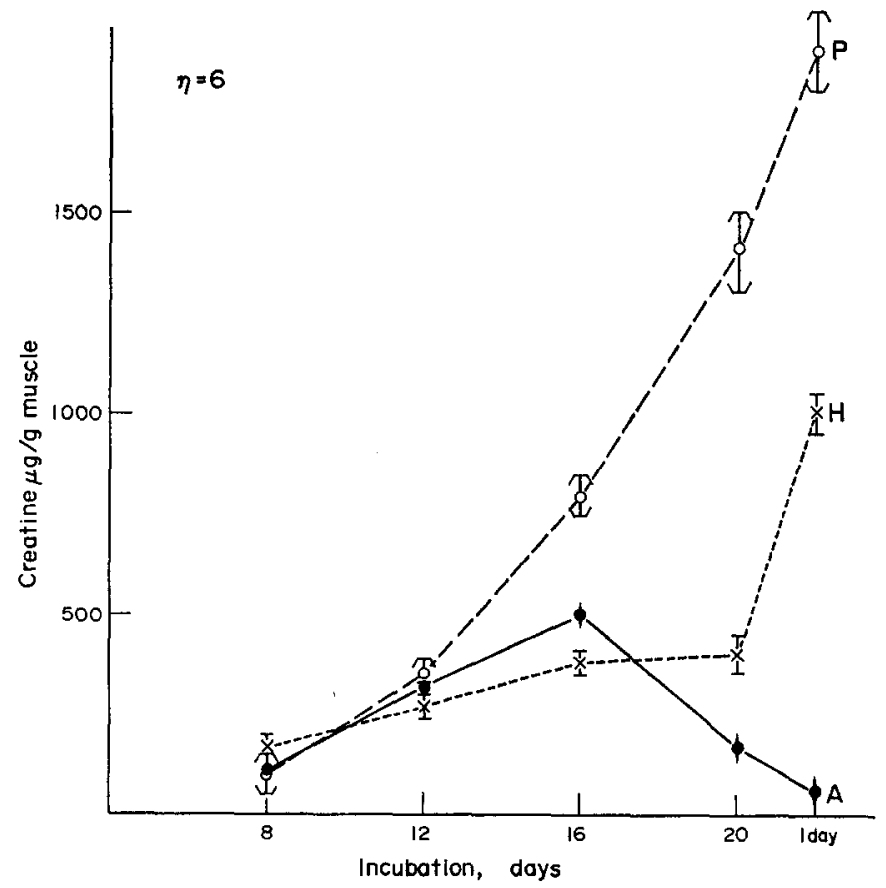

FIG. 7. Quantitative changes in the creatine in the muscles of the chick during prenatal development. 
The levels of creatine and creatinine present a picture of phosphocreatine synthesis since the creatinine synthesis is proportional to the total amount of creatine and $\mathrm{CP}$ in the muscle. Consequently, creatinine cannot be reutilized for any purpose and is therefore excreted (Cantarow \& Schepartz, 1967). The data on creatinine and creatine are therefore collected to evaluate the creatine metabolism in the muscle during development. Figure 7 shows the creatine content of the muscle during development. PLD shows a continuous increase but $\mathrm{H}$ maintains a steady state between the sixteenth and twentieth days. There is a consistent depletion of creatine content in ALD after the sixteenth day. Between the eighth and twelfth day all three muscles contain the same amount of creatine.

The endogenous creatinine content (Fig. 8) presents interesting trends. PLD proportionately increases in creatinine content in contrast to the heart. However, the heart shows an increased level on the twelfth day when compared to the eighth day of incubation. ALD follows the trend of PLD up to the sixteenth day

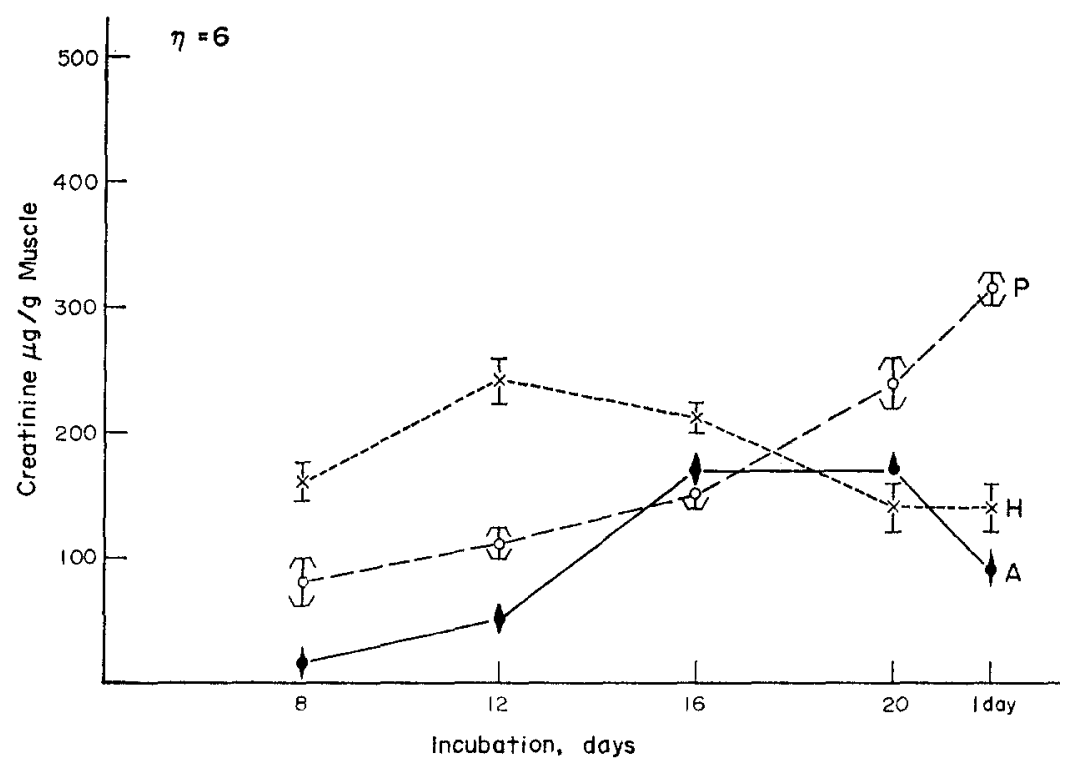

FIG. 8. Quantitative changes in the creatinine in the muscles of the chick during prenatal development.

but maintains the same steady state between the sixteenth and twentieth day of incubation and shows a reducing trend between the twentieth and first day. It is suggested that depleting trends of creatinine may be due to excretion much earlier than ALD (Fig. 8) and PLD never excretes even after the first day of hatching.

Creatine/creatinine ratios (Fig. 9) suggest hydrolysis of creatine to form creatinine. In other words, these ratios serve to indicate the creatine potential in the muscle. In ALD the ratios are gradually reduced in contrast to PLD and the 


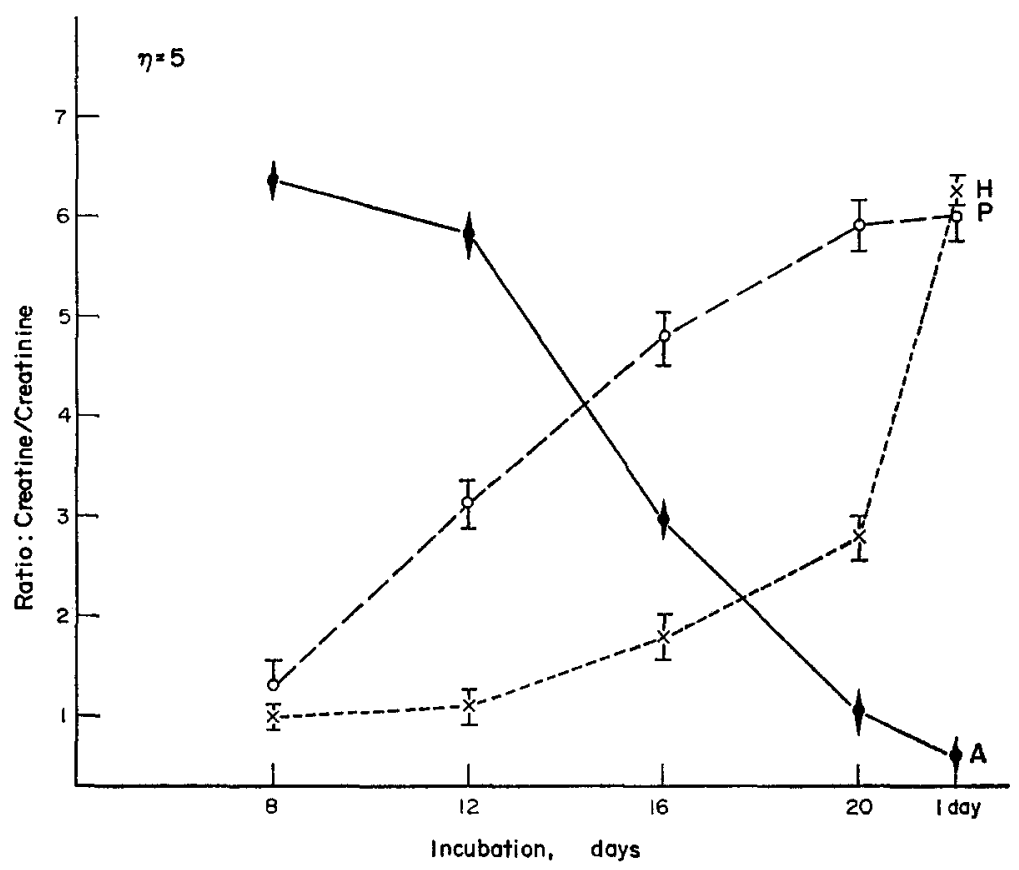

FIG. 9. Relative changes of creatine/creatinine content in the muscles of the chick during prenatal development.

heart. As regards creatine potential, ALD behaves differently from those of PLD and the heart, in that more creatine is converted into creatinine in the former. In PLD and the heart the creatine is synthesized at a rapid rate with the utilization of arginine and glycine in spite of accumulating creatinine. It could be possible that creatine synthesis may be more intense in the developing heart in spite of its excretion (Figs. 8 and 9).

Figure 10 shows the ratios of $\mathrm{CP} /$ creatinine during the development of these muscles. The heart maintains a steady level during the development with reference to these ratios. In the heart the $\mathrm{CP}$ and creatinine follow a negative trend with reference to the days of incubation but the ratios are still at a steady level. It is possible that $\mathrm{CP}$ is being converted into creatinine in the heart muscles. PLD, in contrast to increasing $\mathrm{CP}$ and increasing creatinine, maintains an increase in the $\mathrm{CP} /$ creatinine ratio up to the sixteenth day and thereafter marks a reducing trend. $\mathrm{Up}$ to the sixteenth day more of CP is being converted into creatinine but later on creatine is being converted to creatinine in PLD. Just the opposite trends are discernible in ALD.

Figure 11 explains the conversions of creatine to creatine phosphate and vice versa through their ratios. The larger ratios indicate a $\mathrm{CP}$ breakdown and the lower ratios indicate the phosphorylation of creatine by creatine kinase activity. This is an approximation of the interconversions, but more light can be thrown on 

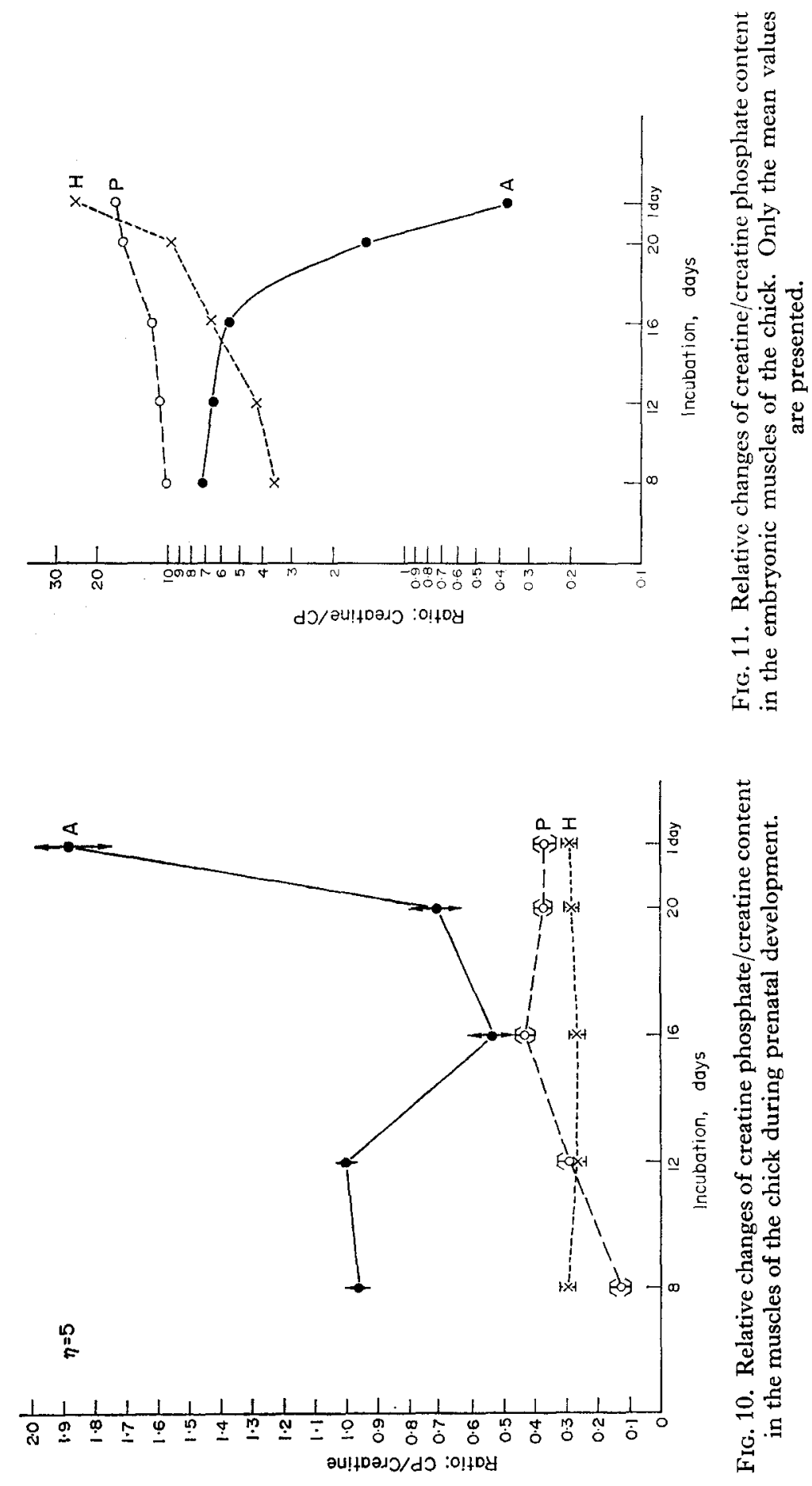
creatine phosphate synthesis by the results of the phosphorylase activity. As is shown in Fig. 11 the trend of the ratios in ALD and PLD is diametrically opposite to each other. The ratio in ALD is very much reduced with an increase in the days of incubation whereas in PLD it increases; the heart tissue also follows a similar trend to PLD. Based on these results it can be suggested that the heart as well as PLD break down CP more efficiently than ALD. It is one of the characteristics of phasic as well as rhythmic muscle such as PLD and the heart respectively to utilize CP during contractions.

In order to test this hypothesis more information is required and also it should be confirmed whether PLD is actively engaged in contractility during embryonic development like the heart. However, one can easily imagine that during the mechanical stimulation of PLD twitches may be produced which may result in the breakdown of CP. On the other hand, ALD being a slow muscle, twitch development may not be possible and the muscle undergoes tetanic contractions. Hence, the CP level is more than that of creatine and, as a result, low ratios were obtained. The progressive rise in the ratios in the PLD and heart and the progressive reduction of the same in ALD show the differentiation of the muscle twitch and tonic activities.

Any biochemical mechanism involving phosphorylations always tends to operate itself at maximum efficiency (Slater, 1966). Maximum efficiency will be obtained if the rate of production of high-energy phosphate equals its rate of utilization (Pardee \& Ingraham, 1960). Self-regulating mechanisms do exist to run phosphorylation at maximum efficiency as in mitochondria (Ernster \& Luft, 1964). In the muscle the transphosphorylation of ATP to CP may be cited as one of the self-regulating mechanisms for immediate utilization of high energy (Huennekens \& Whiteley, 1960). If it is an oxidative phosphorylation, the intramitochondrial ratio $\mathrm{ATP} /(\mathrm{ADP})\left(\mathrm{P}_{\mathrm{i}}\right)$, called phosphate-potential, is considered as the major controlling factor. The same ratio of total ATP and total ADP inorganic phosphate in the present investigation also is considered, to study the efficiency of the phosphorylation mechanisms. Larger ratios in such a case always indicate a high phosphate potential. A greater rate of utilization of ATP for work is represented by low ratios; consequently, $\mathrm{ADP}$ and $\mathrm{PO}_{4}$ levels increase. Figure 12 is the outcome of such a treatment. Ratios in the heart decrease up to the sixteenth day and afterwards markedly increase. In the case of skeletal muscles ALD exhibits a declining ratio with an increase in development and a contrary trend is noticed in PLD. Higher phosphate potential in heart and PLD may be an index for the phasic and rhythmic behaviour of the muscles respectively. Lower ratios in ALD may be due to a less efficient mechanism of phosphorylation.

Figure 13 presents the CP and ATP contents of the muscles. This relationship is presented in the form of the CP/ATP ratio which would indirectly suggest the potential for transphosphorylations. High ratios indicate greater CP levels whereas low ratios indicate a high ATP content. Except in heart, the ALD and PLD show high ratios with an increase in days of incubation. The increase in between 8 and 12 days is the same in ALD and PLD but after the twelfth day the proportion 

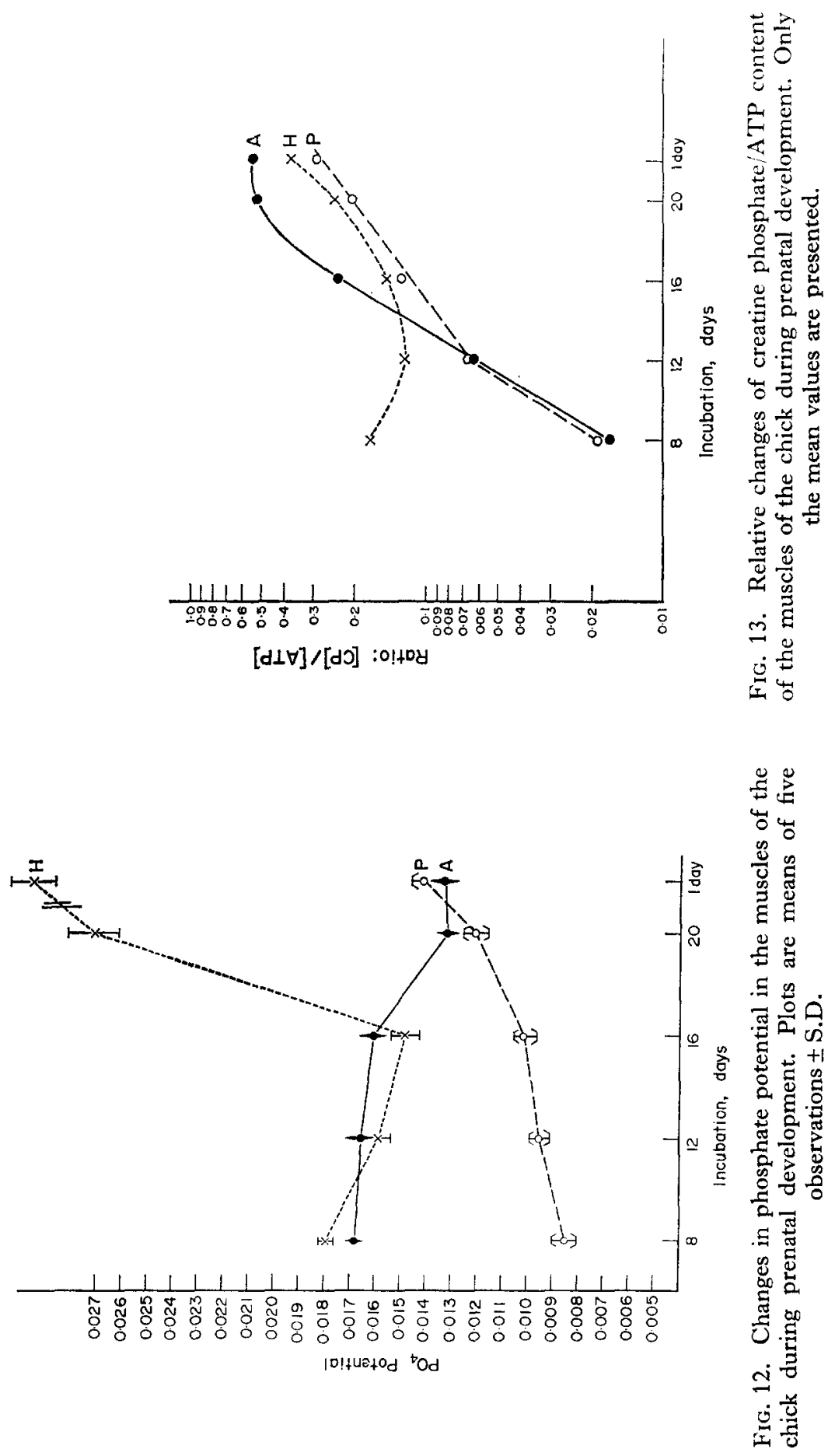
varies, in that the ALD shows a greater increase than does PLD. The heart shows a decrease in this trend up to the twelfth day and then establishes an increase in the same manner as PLD. Between the sixteenth and first day of development the ALD has higher ratios than the heart and PLD whereas PLD has lower ratios. A high ATP content in PLD and the heart as revealed by low ratios may suggest the ability of the muscle for transphosphorylation, since it is the ATP that is hydrolysed for $\mathrm{CP}$ synthesis. The proportionate increase of the ratios in logarithmic progression in ALD may be due to less ATP content which would hydrolyse for $\mathrm{CP}$ synthesis. However, the ability of transphorylations can be assessed better by the study of the ATP-Cr-transphosphorylase activity of the muscle homogenates.

During the early embryonic stages the transphosphorylase activity is poor (Fig. 14). Its activity is gradually increased in any muscle during development and hence greater amounts of CP labile $P_{i}$ are obtained. The specific activity (when expressed as $P_{i}$ phosphorylated per min per $\mathrm{mg}$ homogenate protein) in all three muscles shows an increasing trend with incubation. The specific activity is higher in the heart than in ALD and PLD. The specific activity increases in logarithmic proportion in the heart. Among these two the PLD shows more specific activity

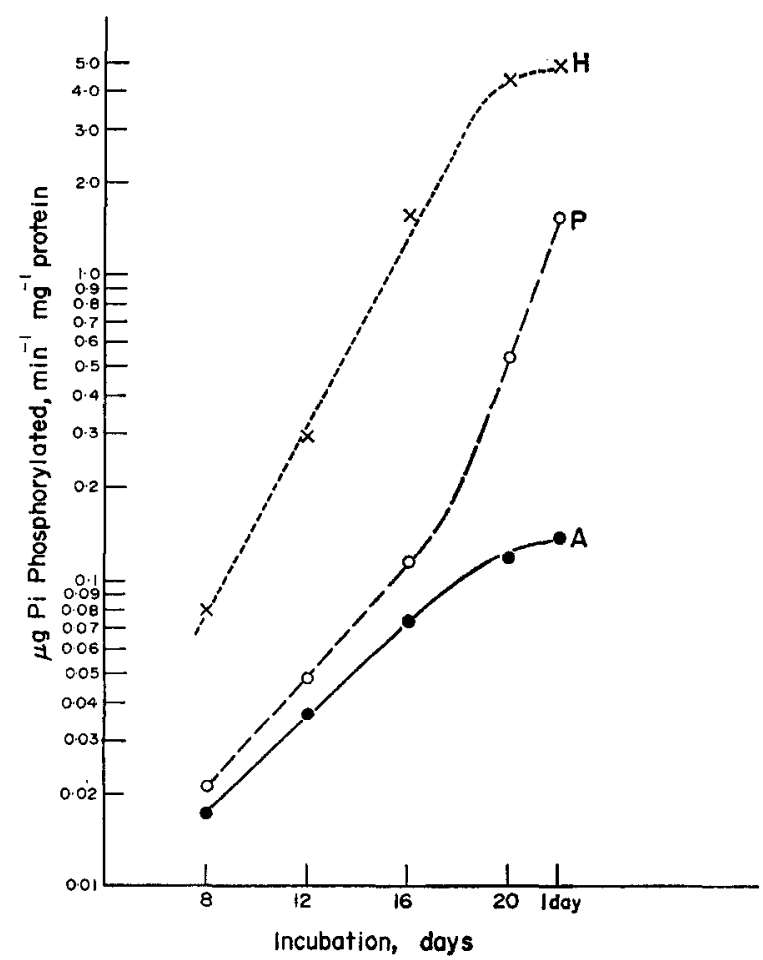

FIG. 14. Changes in the specific ATP-creatine transphosphorylase activity of the enzyme from muscles of the chick during embryonic development. H, Heart; P, PLD; A, ALD. 
than ALD. Up to the sixteenth day the PLD increases the specific activity enormously in logarithmic proportion (Fig. 14). However, in ALD the increase is not as great as in PLD during the later stages of development.

To evaluate the differentiation of contractility in these muscles, besides the phosphate potential, CP potential and the ability of transphosphorylation, an understanding of the myofibrillar ATPase activity is essential since it is the myofibril which utilizes the transphosphorylation scheme for contractility. The results on myofibrillar ATPase are presented in Figs. 15 and 16. In general, myofibrillar ATPase of all three muscles are activated more by $\mathrm{Mg}^{2+}$ than by $\mathrm{Ca}^{2+}$ in contrast to myosin ATPase. $\mathrm{Ca}^{2+}$ activated ATPase follows a reducing trend in all the muscles with an increase in days of incubation (Fig. 15).

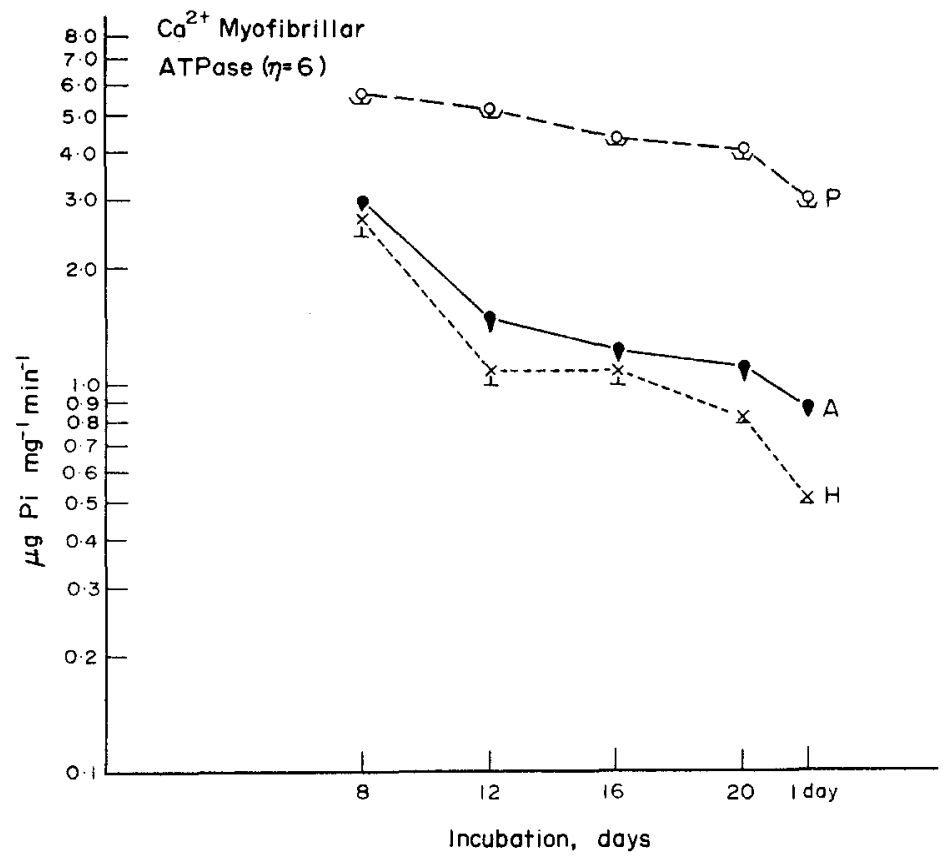

FIG. 15. Changes in the $\mathrm{Ca}^{2+}$ activated myofibrillar ATPase activity of embryonic muscles.

$\mathrm{Ca}^{2+}$ ATPase is greater in PLD than the other two muscles. The heart has a very low ATPase when compared to ALD and PLD. $\mathrm{Mg}^{2+}$ ATPase decreased gradually in ALD up to the twentieth day of incubation and then sharply till the first day after hatching (Fig. 16). The reducing trends in PLD and the heart are seen up to the twentieth day. On the first day after hatching the ATPases show a higher activity in these muscles.

The results of ocular shortening and relaxation are presented in histograms (Fig. 17). In a medium consisting of $0 \cdot 1 \mathrm{M} \mathrm{KCl}$ and $1 \mathrm{mM}$ ATP only ALD shortens whereas the other two muscles relax. The magnitude of relaxation increases 


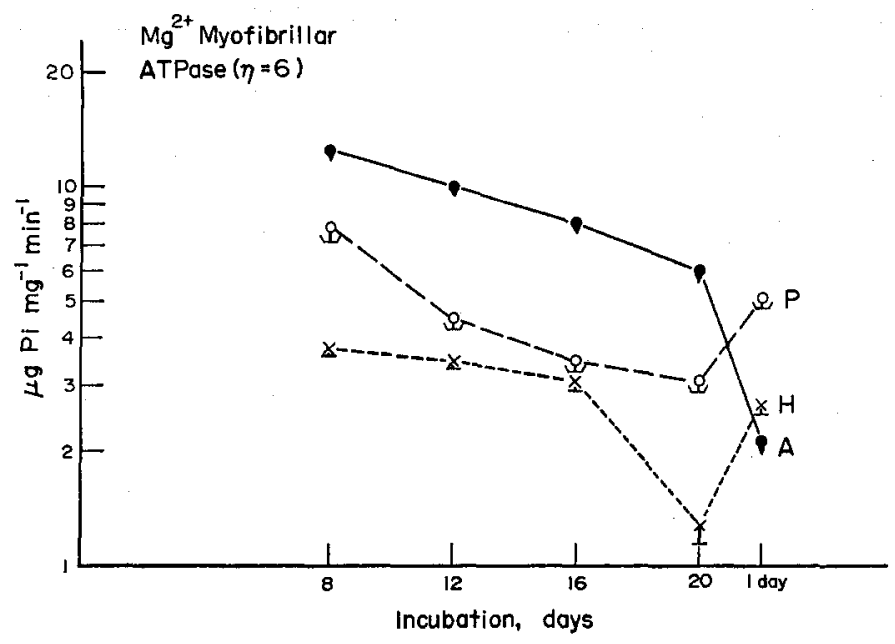

FIG. 16. Changes in the $\mathrm{Mg}^{2+}$ activated myofibrillar ATPase activity in the embryonic muscles.

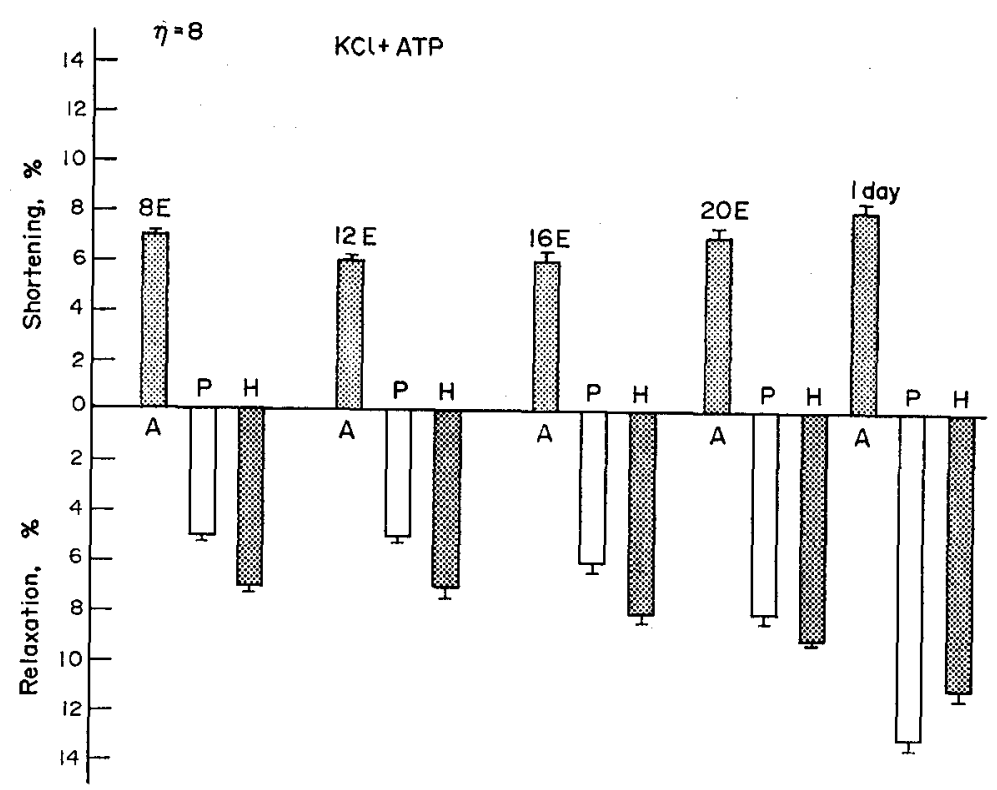

FIG. 17. Histograms representing the percentage shortening and relaxation of glycerol-extracted fibres from the muscles of an embryonic chick at different stages of development. A, ALD; P, PLD; $H$, heart.

in PLD and the heart along with the increase in incubation. Up to the twentieth day the heart fibres are more relaxed than those of PLD but on the first day after hatching the PLD fibres show greater relaxability than those of the heart. The 
extent of shortening in ALD in a medium of $0.1 \mathrm{M} \mathrm{KCl}$ and $1 \mathrm{mM}$ ATP follows a $V$-shaped trend with a minimum shortening between the twelfth and sixteenth day and an increase in shortening at either end of the period of the incubation scale. The ALD fibres are more contractile on the first day than in the earlier stages. The reason for the contractility in ALD fibres and relaxability in the fibres of other muscles might be sought in the $\mathrm{Ca}^{2+}$ binding property. $\mathrm{Ca}^{2+}$ is bound in PLD and heart muscles and even if a trace is present it must have been lost during extraction. The differentiation of the tubular system may be suggested for the $\mathrm{Ca}^{2+}$ binding. It is possible that the heart and PLD differentiate the sarcotubular system more rapidly than ALD. This is further confirmed by the study of the shortening of the fibres in the solution of ATP- $\mathrm{Ca}^{2+}-\mathrm{KCl}$. In the presence of $\mathrm{Ca}^{2+}$ all three fibres contract but the extent of contraction varies with the type of fibre and incubation. In $10 \mathrm{mM} \mathrm{CaCl}{ }_{2}$ the percentage of shortening in PLD fibres gradually increases with an increase in the incubation days, whereas in the heart it shows just the opposite trend. In ALD the maximum shortening occurs on the sixteenth day but on either side of the incubation scale the ocular shortening is decreased and thereby a Gaussian distribution curve is obtained for ALD with $10 \mathrm{mM} \mathrm{Ca}{ }^{2+}$.

These results suggest the sensitivity of the fibre to $\mathrm{Ca}^{2+}$. The PLD fibres become more sensitive to $\mathrm{Ca}^{2+}$ with an increase in the days of incubation whereas the heart fibres lose their sensitivity to $\mathrm{Ca}^{2+}$ with development. The ALD fibres show a hybrid relationship with respect to $\mathrm{Ca}^{2+}$ sensitivity. Up to the sixteenth day ALD increases its sensitivity like that of PLD but afterwards decreases like that of the heart. All these results throw light on the differentiation of the biochemical organization of the fibrillar arrangement in these muscles.

\section{DISCUSSION}

Monroy (1965) reviewed the literature concerning the role of adenine nucleotides in the differentiation of embryonic tissues. Particularly does the ADP level in relation to the ATP level present a picture of metabolic pathways existing in the cell (Mahler \& Cordes, 1966). The ATP level in general is known to decrease with development in all the muscle types (Fig. 1) and the ADP/ATP ratios increase. This evidently shows that the ADP level in the muscle increases with development. It could be suggested that the increased level of ADP followed by a decreased ATP level is due to the active transphosphorylation mechanisms existing in the muscle types; their rates are increased with development. The increase in CP (Fig. 13) and the decrease in $\mathrm{PO}_{4}$ with development support this.

The increase in ADP levels with development occurs besides a uniform increase in $\mathrm{P} / \mathrm{O}$ ratios (Radha, 1972) in the muscle types. An increase in the $\mathrm{P} / \mathrm{O}$ ratio would generally indicate a greater ATP synthesis, but the ATP level in the present study decreases with development. These results indicate that even though the ATP is synthesized at a faster rate (as substantiated by increasing $\mathrm{P} / \mathrm{O}$ ratios) the utilization of it is increased by way of transphosphorylations in the 
muscle types with reference to development. The degree or the extent of these transphosphorylations is different in the muscle types during development. However, the above analysis may not give a correct picture of the rates of ATP yield or utilization.

A different treatment of the data was therefore prompted wherein (Fig. 6) the $\mathrm{PO}_{4}$ potential indicated a different meaning by which the tendency of the tissue to utilize ATP for specific physiological processes is explained. Greater $\mathrm{PO}_{4}$ potential ratios indicate a lower rate of ATP utilization.

Based on the $\mathrm{PO}_{4}$ potential results, ALD during development lowers the ratio and consequently a greater amount of ATP is utilized. The heart muscle resembles ALD up to the sixteenth day and thereafter resembles PLD, where the utilization of ATP is reduced gradually during the development. When all three muscles are compared at each stage of incubation, PLD bears lower ratios than ALD and has a greater utilization of ATP.

The explanation for these trends must be sought in (1) development of energyexpending processes and (2) development of mechanisms involving transphosphorylations.

During intermediate stages ALD is rich in protein compared to PLD (Radha, 1972 ) and, with possibly a higher rate of protein synthesis, meets a greater demand of ATP utilization as revealed by the pattern of the $\mathrm{PO}_{4}$ potential. The pattern of the latter in the heart could be due to a shift in the utilization of ATP after the sixteenth day. The second possibility mentioned above may involve creatine-ATP transphosphorylations. Compared at each period of development (Fig. 12), greater utilization of ATP was recorded in PLD at all stages except on the first day. Transphosphorylase activity concomitantly is very high in PLD, hence the ATP utility may be towards greater synthesis of CP or increased CP content (Fig. 6). The pattern of ATP utility indicates a decrease in PLD and an increase in ALD with reference to development. The ALD is made of fibres of "Felderstruktur" and the PLD is of "fibrillenstruktur" (Ginsborg, 1960; Hess, 1970). Kruger (1950) observed only slow fibres in ALD and fast fibres in PLD by his spontaneous electrical recordings. Several morphological characteristics like the absence of a regular tubular T-system and reduced sarcoplasmic reticulum (Hess, 1970) are often correlated with the function of these muscles.

The T-system acts as a conduction pathway from the outer membrane to the inner SR (Huxley \& Taylor, 1958) where the $\mathrm{Ca}^{2+}$ forms a store for activation and may thus be one of the limiting factors of contractility of these muscles. Another factor is the relaxing factor activity (Hasselbach, 1964; Ebashi \& Endo, 1968), which may, to a greater extent, serve to characterize these muscles. The data on the ocular shortening in $\mathrm{Ca}^{2+}$-deficient medium in ALD may suggest the presence of $\mathrm{Ca}^{2+}$ bound to the proteins but not in the sarcoplasmic reticulum which is not well organized in the slow fibres. Ocular relaxation may be affected in the same manner wherein the absence of $\mathrm{Ca}^{2+}$ and of a high ATP concentration causes the dissociation of (Hasselbach, 1964) contractile protein complex, the acto-myosin. The increasing relaxability is characteristic of the developing PLD and heart fibre. 
$\mathrm{Ca}^{2+}$ ATP medium caused different patterns of shortening in ALD and PLD fibres. The reducing trend in ALD with development may be due to reduced myofibrillar ATPase. The increase in shortening of the PLD fibre may be attributable to the formation (or active synthesis) of troponin molecules, in view of the reduced myofibrillar ATPase activity (Weber, 1966; Ebashi \& Endo, 1968; Ashley, 1970), which channel the energy provided by $\mathrm{Ca}^{2+}$ binding, to trigger the events leading to contraction.

The changes in myofibrillar $\mathrm{Mg}^{2+}$ activated ATPase activity might be associated with changes in the innervation of the muscle during development. However, no direct correlation between acetylcholinesterase activity and ATPases was established (Holland \& Perry, 1969), but attempts have been made by many (George \& Berger, 1966) to show that AchE activity might give an index of the development of the innervation in the muscle. Radha \& Krishnamoorthy (1973) studied the changes in acetylcholinesterase activity of the embryonic muscle homogenates during development. The PLD muscle showed greater activity than the heart and ALD. The results of such a survey presented the possible formation of motor end plates in PLD on the sixteenth day and stabilization of activity in heart from the eighth day and in ALD from the sixteenth day. The present results on $\mathrm{Mg}^{2+}$ myofibrillar ATPase give some indication of a direct correlation of acetylcholinesterase activity with the former.

Acknowledgements-We are grateful to Professor K. Pampapathi Rao for critically reading the manuscript and offering valuable suggestions. We wish to thank Professor C. L. Prosser (University of Illinois, U.S.A.) and Professor B. C. Abbott (University of Southern California, U.S.A.) for their very useful suggestions and encouragement.

\section{REFERENCES}

AsHLEy C. C. (1970) An estimate of calcium concentration changes during the contraction of single muscle fibres. Y. Physiol. 210, 133.

BARANY M. (1967) ATPase activity of myosin correlated with speed and muscle shortening. J. genet. Physiol. 50, 197-216.

Cantarow A. \& Schepartz B. (1967) Biochemistry. Saunders, Philadelphia.

Davis J. R. \& HaRRIS R. N. (1963) Rapid colorimetric determination of adenine compounds. Analyt. Biochem. 5, 64-69.

Ebashi S. \& Endo M. (1968) Calcium ion and muscle contraction. Prog. Biophys. molec. Biol. $18,123-183$.

ENNOR A. H. (1957) Determination and preparation of N-phosphates of biological origin-I. Phosphocreatine. In Methods in Enzymology (Edited by Colowick S. P. \& KaPLAN N. O.), Vol. III, pp. 850-861. Academic Press, New York.

ENNOR A. H. \& Rosenberg H. (1952) The determination and distribution of phosphocreatine in animals. Biochem. $.51,606-610$.

ERNSTER L. \& LufT R. (1964) Mitochondrial respiratory control: biochemical, physiological and pathological aspects. Adv. Metabol. Disorders 1, 95-100.

Fiske C. H. \& SubBa Row Y. (1925) The colorimetric determination of phosphorus. $\mathcal{F}$. biol. Chem. 66, 375-400.

George J. C. \& Berger A. J. (1966) Avian Myology. Academic Press, New York.

GINSBORG B. L. (1960) Some properties of avian skeletal muscle fibres with multiple neuromuscular junctions. F. Physiol., Lond. 154, 581-598. 
GlIck D. E. (1954) Methods in Biochemical Analysis, Vol. I. Interscience, New York.

Guth L. L. (1969) Neurosciences research program Bulletin 7, 1-73. M.I.T. Press, Massachusetts Institute of Technology, Cambridge, U.S.A.

Hasselbach W. (1964) Relaxing factor and the relaxation of muscle. Prog. Biophys. Chem. $14,167-222$.

Hess A. (1970) Vertebrate slow muscle fibres. Physiol. Rev. 50, 40-62.

Holland D. L. \& Perry S. V. (1969) The adenosine triphosphatase and calcium iontransporting activitp of the sarcoplasmic reticulum of developing muscle. Biochem. $\mathcal{F}$. 114, 161-170.

Huennekens F. M. \& Whiteley H. R. (1960) Phosphoric acid anhydrides and other energy rich compounds. In Comparative Biochemistry (Edited by FloRKIN M. \& MAsON H. S.). Academic Press, New York.

HuXIEY A. F. \& TAYLoR R. E. (1958) Local activation of striated muscle fibres. F. Physiol. Lond. 144, 426-443.

Kruger P. (1950) Untersuchungen am Vogelflugel. Zool. Anzeig 145, 445-460.

LAYNE D. (1957) Spectrophotometric and turbidimetric methods for measuring proteins. In Methods in Enzymology (Edited by Colowick S. P. \& KaPlaN N. O.), Vol. III, p. 454. Academic Press, New York.

MAHLer H. R. \& CoRdes E. H. (1966) Biological Chemistry. Harper International edition. Harper and Row, New York.

Milhorat A. T. (1967) Exploratory concepts in muscular dystrophy and related disorders. Excerpta. Med. Found., Amsterdam.

MonRoy A. (1965) Biochemical Aspects of Fertilization. In The Biochemistry of Animal Development (Edited by Weber R.), Vol. I, pp. 73-139. Academic Press, New York.

Noda L., Kuby S. \& LARDY H. (1955) ATP-creatine transphosphorylase. In Methods in Enzymology (Edited by Colowick S. P. \& Kaplan N. O.), Vol. II, pp. 605-610. Academic Press, New York.

OSER B. L. (Ed.) (1965) Hawk's Physiological Chemistry, 13th Edition. McGraw-Hill, New York.

Pardee A. B. \& Ingraham L. L. (1960) Free energy and entropy in metabolism. In Metabolic Pathways (Edited by Greenberg M.), Vol. I. Academic Press, New York.

PerRY S. V. (1951) The adenosinetriphosphate activity of myofibrils isolated from skeletal muscle. Biochem. F. 48, 257-265.

PERRY S. V. (1952) The bound nucleotide of the isolated myofibril. Biochem. F. 51, 495-499.

PERRY S. V. (1955) Myosin adenosine triphosphatase activity. In Methods in Enzymology (Edited by Colowick S. P. \& Kaplan N. O.), Vol. II, pp. 582-588. Academic Press, New York.

RADHA E. (1972) Biochemical and metabolic studies of the fast, slow and cardiac muscles of chick during development. Unpublished thesis.

RAdHA E. \& KRISHNAMOORTHY R. V. (1973). Acetylcholine esterase activity in the embryonic muscles of chick. Curr. Sci. 42, 26-27.

Ruegg J. C., Schadler M., Steiger G. J. \& Muller G. (1971) Effects of inorganic phosphate on contractile mechanism. Pfligers Arch. ges. Physiol. 325, 359-364.

Slater E. C. (1966) Oxidative Phosphorylation. In Comprehensive Biochemistry (Edited by Florkin M. \& Stotz E. H.), Vol. 14. Elsevier, Amsterdam.

WEBER A. (1966) Energized calcium transport and relaxing factors. In Current Topics in Bioenergetics (Edited by Sanadi D. R.), Vol. I, pp. 203-254. Academic Press, New York.

Key Word Index-Anterior latissimus dorsi; posterior latissimus dorsi; creatine transphosphorylase; creatine phosphate; phosphate potential; creatinine; creatine; myofibrillar ATPase; glycerinated fibre; ocular shortening; molecular differentiation. 